\title{
Serum metabolites detect the presence of advanced fibrosis in derivation and validation cohorts of patients with non-alcoholic fatty liver disease
}

\author{
Cyrielle Caussy, ${ }^{1,2}$ Veeral H Ajmera, ${ }^{1}$ Puneet Puri, ${ }^{3}$ Cynthia Li-Shin Hsu, ${ }^{\oplus} 1$ \\ Shirin Bassirian, ${ }^{1}$ Mania Mgdsyan, ${ }^{1}$ Seema Singh, ${ }^{1}$ Claire Faulkner, ${ }^{1}$ Mark A Valasek, ${ }^{4}$ \\ Emily Rizo, ${ }^{1}$ Lisa Richards, ${ }^{1}$ David A Brenner, ${ }^{1,5}$ Claude B Sirlin, ${ }^{6}$ Arun J Sanyal, ${ }^{3}$ \\ Rohit Loomba ${ }^{1,5,7}$
}

\begin{abstract}
${ }^{1}$ NAFLD Research Center, Department of Medicine, La Jolla, California, USA ${ }^{2}$ Université Lyon 1, Hospices Civils de Lyon, Lyon, California, France

${ }^{3}$ Virginia Commonwealth University, Richmond, Virginia, USA

${ }^{4}$ Department of Pathology, University of California at San Diego, La Jolla, California, USA Division of Gastroenterology, Department of Medicine, La Jolla, California, USA

${ }^{6}$ Liver Imaging Group, Department of Radiology, University of California, San Diego, La Jolla, California, USA ${ }^{7}$ Division of Epidemiology, Department of Family and Preventive Medicine, University of California at San Diego, La Jolla, California, USA
\end{abstract}

Correspondence to Professor Rohit Loomba, Division of Gastroenterology and Epidemiology, University of California at San Diego, La Jolla 92093-0063, US roloomba@ucsd.edu

CC, VHA and PP contributed equally.

Received 13 September 2018 Revised 22 October 2018 Accepted 29 November 2018 Published Online First 19 December 2018

Check for updates

(C) Author(s) (or their employer(s)) 2019. № commercial re-use. See rights and permissions. Published by BMJ.

To cite: Caussy $C$ Ajmera VH, Puri P, et al. Gut 2019;68:1884-1892.

\section{ABSTRACT}

Objective Non-invasive and accurate diagnostic tests for the screening of disease severity in non-alcoholic fatty liver disease (NAFLD) remain a major unmet need. Therefore, we aimed to examine if a combination of serum metabolites can accurately predict the presence of advanced fibrosis.

Design This is a cross-sectional analysis of a prospective derivation cohort including 156 well-characterised patients with biopsy-proven NAFLD and two validation cohorts, including (1) 142 patients assessed using MRI elastography (MRE) and(2) 59 patients with biopsy-proven NAFLD with untargeted serum metabolome profiling.

Results In the derivation cohort, 23 participants (15\%) had advanced fibrosis and 32 of 652 analysed metabolites were significantly associated with advanced fibrosis after falsediscovery rate adjustment. Among the top 10 metabolites, 8 lipids (5alpha-androstan-3beta monosulfate, pregnanediol3-glucuronide, androsterone sulfate, epiandrosterone sulfate, palmitoleate, dehydroisoandrosterone sulfate, 5alphaandrostan-3beta disulfate, glycocholate), one amino acid (taurine) and one carbohydrate (fucose) were identified. The combined area under the receiver operating characteristic curve (AUROC) of the top 10 metabolite panel was higher than FIB--4 and NAFLD Fibrosis Score (NFS) for the detection of advanced fibrosis: 0.94 (95\% Cl 0.897 to 0.982 ) versus 0.78 ( $95 \%$ Cl0.674 to 0.891$), \mathrm{p}=0.002$ and versus 0.84 ( $95 \% \mathrm{Cl} 0.724$ to 0.929 ), $p=0.017$, respectively. The AUROC of the top 10 metabolite panel remained excellent in the independent validation cohorts assessed by MRE or liver biopsy: c-statistic of 0.94 and 0.84 , respectively.

Conclusion A combination of 10 serum metabolites demonstrated excellent discriminatory ability for the detection of advanced fibrosis in an derivation and two independent validation cohorts with greater diagnostic accuracy than the FIB-4-index and NFS. This proof-ofconcept study demonstrates that a non-invasive bloodbased diagnostic test can provide excellent performance characteristics for the detection of advanced fibrosis.

\section{INTRODUCTION}

Non-alcoholic fatty liver disease (NAFLD) is currently recognised as one of the most prevalent aetiologies of chronic liver disease worldwide and an increasingly common cause of cirrhosis and hepatocellular carcinoma. ${ }^{12}$ NAFLD exists as a

\section{Significance of this study}

What is already known on this subject?

- FIB-4 and NAFLD Fibrosis Score (NFS) are clinical prediction rules that provide high accuracy for prediction of the presence of advanced fibrosis in NAFLD.

- FIB-4 and NFS lack specificity and leave many patients with indeterminate results; therefore, more accurate blood tests are needed.

What are the new findings?

- In patients with biopsy-proven NAFLD, a serum metabolomics panel was able to predict the presence of advanced fibrosis with high diagnostic accuracy and outperformed FIB-4 and NFS.

- The serum metabolomics panel maintained excellent diagnostic accuracy and superiority over FIB-4 in two validation cohort of patients with and without NAFLD.

How might it impact on clinical practice in the foreseeable future?

- A blood-based serum metabolite panel can accurately diagnose advanced fibrosis with greater diagnostic accuracy than FIB-4 and NFS and may be a useful tool to screen at-risk patients for advanced disease.

spectrum ranging from non-alcoholic fatty liver (NAFL), which is generally considered to be at low risk of disease progression, to non-alcoholic steatohepatitis (NASH) with or without fibrosis. ${ }^{3}$ Multiple studies have demonstrated that fibrosis stage is the most important predictor of morbidity and mortality. ${ }^{3-5}$

Although liver biopsy is considered as the gold standard for the diagnosis of liver fibrosis and $\mathrm{NASH}$, it is impractical to use for the estimated 80-100 million adults with NAFLD in the USA Likewise, the most accurate biomarkers currently available are imaging-based modalities which are not available to many primary care providers and community gastroenterologists. ${ }^{6}$ Therefore, there is an urgent need for non-invasive, easy-to-perform 
and widely available diagnostic alternatives to assess the disease severity. Several non-invasive tests have been developed for the detection of advanced fibrosis and $\mathrm{NASH}^{7}$ including clinical predictive rules combining serum markers and clinical variables such as the commonly used FIB-4 index. ${ }^{8}$ However, none of the currently available biomarkers are able to differentiate NASH from simple steatosis or stage liver fibrosis with high sensitivity and specificity. ${ }^{9}$

In recent years, metabolome profiling has provided new insights into the molecular signature of diseases including NAFLD. Several studies have identified specific metabolomic profiles associated with different stages of disease in NAFLD. ${ }^{10-17}$ Significant changes in key pathways including bile acids, ${ }^{18}$ amino acids, ${ }^{13-15} 17$ steroids hormones ${ }^{16} 1920$ and fatty acids ${ }^{10} 1421$ have been reported in subjects with NAFLD, making this an appealing target for future diagnostics. ${ }^{11}$ Indeed, the changes in these metabolites are likely to reflect specific pathways of liver injury related to NASH or advanced fibrosis making them compelling biomarkers. Thus, we hypothesised that a combination of metabolites could be a more accurate diagnostic test than available clinical predictive rule such as FIB-4 index and NAFLD Fibrosis Score (NFS) for the detection of advanced fibrosis and could accurately diagnose the presence of NASH.

Using a well-characterised prospective cohort of patients with biopsy-proven NAFLD, we aimed to investigate whether a combination of serum metabolites associated with advanced fibrosis could accurately predict its presence with better diagnostic accuracy than widely used clinical prediction rules. We then validated the diagnostic performance of the combination of serum metabolites for the detection of advanced fibrosis in two independent validation cohorts of prospectively recruited individuals with advanced fibrosis assessed by MRI elastography (MRE) in one cohort and liver biopsy in the second validation cohort.

\section{MATERIALS AND METHODS}

\section{Study participants and design of the biopsy-proven NAFLD derivation cohort}

This was a cross-sectional analysis of a discovery cohort that included 156 participants prospectively recruited between October 2011 and May 2014 at the University of California at San Diego (UCSD) NAFLD Research Center. ${ }^{22-25}$ All patients with suspected NAFLD with a clinical indication for liver biopsy underwent a careful evaluation for other causes of hepatic steatosis and liver disease through a standardised research visit including detailed medical and alcohol use history as well as anthropometric and physical examination. The study was performed according to STARD criteria listed in online supplementary table 1.

\section{Inclusion criteria and exclusion criteria of the NAFLD biopsy- proven derivation cohort}

Participants were included in the study if they were 18 years or older with suspected NAFLD and were willing and able to provide informed consent.

Participants were excluded if they met any of the following criteria: history of regular and excessive alcohol consumption within 2 years of recruitment ( $\geq 14$ drinks/week for men or $\geq 7$ drinks/week for women); use of hepatotoxic drugs or drugs known to cause hepatic steatosis; and any evidence of secondary causes of hepatic steatosis or concomitant liver disease. Please see online supplemental material for detailed inclusion and exclusion criteria.

\section{Study participants and design of the validation Twin and} Family Cohort

This was a cross-sectional analysis of a prospective cohort study of patients from the Twin and Family Study (ClinicalTrials.gov: NCT01643512) residing in Southern California. This study included a total of 142 participants. All participants underwent a standardised clinical research visit including detailed medical history, alcohol quantification using Skinner and Audit questionnaire, anthropometric exam, physical exam and biochemical testing at the UCSD NAFLD Research Center ${ }^{23} 26-29$ between December 2011 and January 2014. NAFLD was assessed clinically and quantified by MRI proton density fat fraction (MRI-PDFF) and liver fibrosis was assessed by MRE at the MR3T Research Laboratory. Research visits and imaging procedures were performed the same day for each pair of twins, parent-offspring or siblings.

\section{Inclusion and exclusion criteria of the validation Twin and Family Cohort}

Patients were included if they were twins, siblings or parent-offspring at least 18 years of age, willing and able to complete all research procedures and observations. Participants were excluded from the study if they met any of the following criteria: significant alcohol intake $(>10 \mathrm{~g} /$ day in females or $>20 \mathrm{~g} /$ day in males) for at least three consecutive months over the previous 12 months or if the quantity of alcohol consumed could not be reliably ascertained; clinical or biochemical evidence of liver diseases other than NAFLD. Please see online supplemental material for detailed inclusion and exclusion criteria.

\section{Study participants and design of the validation biopsy-proven NAFLD cohort}

Patients with suspected NAFLD were prospectively recruited from fatty liver disease and primary care clinics at Virginia Commonwealth University. Patients underwent detailed history and laboratory evaluation. Liver biopsy was performed on subjects with suspected NAFLD and a fasting serum sample was obtained within 90 days of the liver biopsy. Liver histology was assessed by two dedicated hepato-pathologists using the NASH CRN scoring system.

\section{Inclusion and exclusion criteria of the validation biopsy proven NAFLD cohort}

This study included consenting adults with biopsy-proven NAFLD and excluded patients with alcohol intake $>20 \mathrm{~g} /$ day (women) and $>30 \mathrm{~g} /$ day (men), medications known to cause steatosis or other causes of liver disease.

\section{Untargeted metabolome profiling}

Serum metabolite assessment was performed by Metabolon (Durham, North Carolina, USA). Samples were extracted and split into equal parts for analysis on the GC/MS and LC/MS/ MS platforms. ${ }^{30}$ Software was used to match ions to an in-house library of standards for metabolite identification and for metabolite quantitation by peak area integration. ${ }^{31} \mathrm{~A}$ number of internal standards were added to each experimental and process standard sample just prior to injection into the mass spectrometers. A measure of the platform variability was determined by calculating the median relative SD for these internal standards. Profiling of samples on the lipidomics platform was also carried out and data were merged with results from the global profiling platform. Details regarding serum metabolite data acquisition 
have been described in previously ${ }^{23}$ and are available in online supplemental material

\section{MRI assessment}

MRI was performed at the UCSD MR3T Research Laboratory using the 3T research scanner (GE Signa EXCITE HDxt; GE Healthcare, Waukesha, Wisconsin, USA) with all participants in the supine position. MRI-PDFF was used to measure hepatic steatosis, and MRE was used to measure hepatic fibrosis. The details of the MRI protocol have been previously described in references $^{32} 33$ and are detailed in online supplemental material

\section{Histological evaluation in USCD NAFLD cohort and biopsy- proven NAFLD validation cohort}

Histological assessment of liver biopsies was performed by an experienced liver pathologist who was blinded to the patient's clinical and radiological data. Histological scoring was done using the Nonalcoholic Steatohepatitis Clinical Research Network Histologic Scoring System $^{34}$ in which hepatic fibrosis was scored on a scale from 0 to $4(0,1,2,3,4)$, with stage $3-4$ signifying advanced fibrosis, hepatic steatosis and lobular inflammation were scored from 0 to $3(0,1,2$, 3 ), and hepatic ballooning was scored from 0 to $2(0,1,2)$. Steatosis, lobular inflammation and hepatocyte ballooning scores were summed to obtain the NAFLD activity score, which ranged from 0 to 8. Diagnosis of NASH was classified as definite NASH, NAFLD not NASH or 'borderline' NASH. Definite and borderline NASH cases were combined and compared with NAFLD not NASH. These categories were assigned prior to conducting statistical analyses.

\section{Clinical predictive rule}

Age, aspartate aminotransferase (AST), alanine aminotransferase (ALT) and platelet count were used to calculate the FIB-4 index using the formula described previously. ${ }^{35}$ Age, body mass index (BMI), impaired fasting glucose or presence of diabetes mellitus AST, ALT, platelet count and albumin were used to calculate the NAFLD Fibrosis Score using the formula described previously. ${ }^{36}$

\section{Primary and secondary outcomes}

The primary outcome was the presence of advanced fibrosis as defined by histological stage of fibrosis 3-4 in the derivation cohort and biopsy-proven validation cohort and by MRE $\geq 3.63 \mathrm{kPa}$ in the validation cohort.

The secondary outcome was the presence of NASH (borderline and definite $\mathrm{NASH}$ ) versus not NASH histology which could only be tested in the derivation and biopsy-proven NAFLD cohort.

\section{Statistical analysis}

\section{Data preparation}

Unnamed metabolites (325 out of 1046 in the derivation cohort and 411 out of 1181 in the validation cohort) were excluded from the analysis. Metabolites for which there were $>50 \%$ missing values, most likely due to levels below the limits of detection (69 out of 721 in the derivation cohort and 57 out of 770 in the validation cohort), were also excluded from the analysis. For the remaining serum metabolites with $<50 \%$ missing values, values were imputed to half of the observed minimum value for each metabolite assuming that the metabolite was under the limit of detection (online supplemental figure 1). All the data were log-transformed prior to statistical analysis.

\section{Data analysis}

Patients' demographic, anthropometric, clinical and biochemical characteristics were summarised. Categorical variables were shown as counts and percentages, and associations were tested using a $\chi^{2}$ test or Fisher's exact test. Normally distributed continuous variables were shown as mean $( \pm S D)$, and differences between groups were analysed using a two-independent-samples t-test or Wilcoxon-Mann-Whitney test.

Statistical comparison of serum metabolites between advanced fibrosis versus non-advanced fibrosis and NASH versus not NASH histology were assessed using Welch's t-tests. To account for multiple comparisons, the Benjamini-Hochberg false discovery rate (FDR)-adjusted threshold for statistical significance was calculated for the association between serum metabolites and specified outcomes. The 10 metabolites with the strongest association with the specified outcome were included in a logistic regression model, and model performance was evaluated by calculating the area under the receiver operating characteristic curve (AUROC). Ten metabolites were chosen to balance diagnostic accuracy with the number of events per predictor in the logistic regression models. Larger numbers of predictors either failed to improve the diagnostic accuracy or prevented model convergence. For the detection of advanced fibrosis, the top 10 serum metabolite panel was compared with the AUROC for the FIB-4 index and NFS in both the derivation and validation cohorts using the method proposed by DeLong. ${ }^{37}$ In addition, we explored a model of readily available clinical factors that were associated with advanced fibrosis in the derivation cohort derived by backward stepwise elimination with $p<0.05$ as the criterion for inclusion. We also performed exploratory analysis evaluating the impact of MRI proton density fat fraction (MRI-PDFF) measurement on diagnostic accuracy. All statistical analyses were performed using STATA (StataCorp) and SPSS (IBM).

\section{Sample size estimation}

This is a pilot proof-of-concept study including 156 participants with biopsy-proven NAFLD (133 individuals without advanced fibrosis (stage 0-2) and 23 with advanced fibrosis (stage 3-4)) in the derivation cohort. We were able to detect clinically and significantly meaningful difference between the subpopulations.

\section{RESULTS}

\section{Baseline characteristics of the derivation cohort}

In total, 156 patients with biopsy-proven NAFLD were included in this analysis. Participants were a mean age of 49.8 years, $45.5 \%$ non-Hispanic white, predominantly female $(58.3 \%)$ and obese $\left(\right.$ mean $\mathrm{BMI}=32.0 \mathrm{~kg} / \mathrm{m}^{2}$ ). Detailed study cohort flow chart is provided in online supplemental figure 1 . The majority of patients, $87 \%$, had definite or borderline NASH and $15 \%$ had advanced fibrosis. Patients with advanced fibrosis were more likely to be older, Hispanic, have higher BMI, AST, alkaline phosphatase, gamma-glutamyl transferase, haemoglobin $\mathrm{A} 1 \mathrm{c}$ and international normalised ratio. In addition, they had lower platelet count and more hepatocyte ballooning and definite NASH histology (table 1).

\section{Serum metabolites associated with advanced fibrosis in the derivation cohort}

Among the 652 serum metabolites analysed, 32 serum metabolites met the FDR-adjusted threshold for statistically significant association with the presence of advanced fibrosis. These serum metabolites belonged to six super pathways: amino acid, carbohydrate, cofactors and vitamins, lipid, nucleotide and peptide (online supplemental table 2). The fold change of the top 10 serum metabolites associated with the presence of advanced fibrosis is shown in figure 1. 
Table 1 Baseline characteristics of the derivation biopsy-proven NAFLD cohort

\begin{tabular}{|c|c|c|c|c|}
\hline Characteristics & All $(n=156)$ & $\begin{array}{l}\text { No advanced } \\
\text { fibrosis (stage 0-2) } \\
(n=133)\end{array}$ & $\begin{array}{l}\text { Advanced } \\
\text { fibrosis (stage } 3-4) \\
(n=23)\end{array}$ & P value* \\
\hline \multicolumn{5}{|l|}{ Demographics } \\
\hline Age, years & $49.8 \pm 14.3$ & $48.7 \pm 14.1$ & $56.2 \pm 13.9$ & 0.023 \\
\hline Male, n (\%) & $65(41.7)$ & $59(44.4)$ & $6(26.1)$ & 0.114 \\
\hline White, n (\%) & $71(45.5)$ & $65(48.9)$ & $6(26.1)$ & 0.058 \\
\hline Hispanic or Latino, n (\%) & $49(32.7)$ & $36(27.1)$ & $13(39.4)$ & 0.007 \\
\hline $\mathrm{BMI}, \mathrm{kg} / \mathrm{m}^{2}$ & $32.0 \pm 6.0$ & $31.3 \pm 5.0$ & $35.9 \pm 9.5$ & 0.015 \\
\hline \multicolumn{5}{|l|}{ Clinical } \\
\hline Type 2 diabetes, n (\%) & $41(26.3)$ & $32(24.1)$ & $9(39.1)$ & 0.189 \\
\hline \multicolumn{5}{|l|}{ Biological data } \\
\hline AST (U/L) & $42.2 \pm 34.0$ & $38.7 \pm 31.6$ & $62.3 \pm 40.7$ & 0.001 \\
\hline ALT (U/L) & $59.0 \pm 57.0$ & $56.8 \pm 55.0$ & $71.7 \pm 67.1$ & 0.574 \\
\hline Alk P (U/L) & $77.5 \pm 28.3$ & $74.2 \pm 24.6$ & $96.6 \pm 39.4$ & 0.001 \\
\hline GGT (U/L) & $55.5 \pm 51.0$ & $50.6 \pm 48.3$ & $84.7 \pm 57.5$ & 0.001 \\
\hline Total bilirubin (mg/dL) & $1.7 \pm 15.3$ & $0.49 \pm 0.24$ & $0.64 \pm 0.66$ & 0.293 \\
\hline Direct bilirubin (mg/dL) & $0.14 \pm 0.15$ & $0.14 \pm 0.15$ & $0.16 \pm 0.15$ & 0.539 \\
\hline Albumin (g/dL) & $4.46 \pm 0.44$ & $4.49 \pm 0.44$ & $4.27 \pm 0.38$ & 0.019 \\
\hline Glucose (mg/dL) & $106.2 \pm 33.1$ & $104.0 \pm 27.0$ & $118.7 \pm 56.3$ & 0.607 \\
\hline Haemoglobin A1c & $6.0 \pm 0.9$ & $5.97 \pm 0.86$ & $6.48 \pm 1.11$ & 0.048 \\
\hline HOMA-IR & $8.5 \pm 17.3$ & $8.79 \pm 18.51$ & $6.41 \pm 4.96$ & 0.229 \\
\hline Insulin (U/mL) & $34.2 \pm 74.9$ & $35.0 \pm 81.1$ & $30.1 \pm 20.9$ & 0.561 \\
\hline Triglycerides (mg/dL) & $159.0 \pm 83.5$ & $161.5 \pm 82.8$ & $144.7 \pm 87.7$ & 0.397 \\
\hline Total cholesterol (mg/dL) & $182.8 \pm 44.3$ & $184.7 \pm 44.9$ & $171.8 \pm 40.1$ & 0.171 \\
\hline HDL-cholesterol (mg/dL) & $48.7 \pm 15.3$ & $48.7 \pm 15.5$ & $48.3 \pm 14.7$ & 0.888 \\
\hline LDL-cholesterol (mg/dL) & $105.3 \pm 34.6$ & $106.8 \pm 34.6$ & $96.8 \pm 33.7$ & 0.212 \\
\hline Platelet count $\left(10^{3} / \mu \mathrm{L}\right)$ & $244.5 \pm 782.8$ & $255.2 \pm 74.6$ & $195.4 \pm 59.7$ & 0.0001 \\
\hline INR & $1.01 \pm 0.08$ & $1.00 \pm 0.08$ & $1.07 \pm 0.10$ & 0.009 \\
\hline Ferritin (ng/mL) & $190.4 \pm 171.9$ & $200.4 \pm 180.9$ & $135.3 \pm 95.8$ & 0.201 \\
\hline \multicolumn{5}{|l|}{ Clinical prediction rule } \\
\hline FIB-4 & $1.35 \pm 1.24$ & $1.13 \pm 0.87$ & $2.65 \pm 1.97$ & $<0.001$ \\
\hline NAFLD Fibrosis Score & $-1.85 \pm 1.62$ & $-2.160 \pm 1.437$ & $-0.032 \pm 1.755$ & $<0.001$ \\
\hline \multicolumn{5}{|l|}{ Histology } \\
\hline Fibrosis, n (\%) & & & & $<0.001$ \\
\hline 0 & $66(42.3)$ & $66(49.6)$ & $0(0.0)$ & \\
\hline 1 & $51(32.7)$ & $51(38.3)$ & $0(0.0)$ & \\
\hline 2 & $16(10.3)$ & $16(12.0)$ & $0(0.0)$ & \\
\hline 3 & $13(8.3)$ & $0(0.0)$ & $13(56.5)$ & \\
\hline 4 & $10(6.4)$ & $0(0.0)$ & $10(43.5)$ & \\
\hline Steatosis, n (\%) & & & & 0.176 \\
\hline 0 & $3(1.9)$ & $3(2.2)$ & $0(0)$ & \\
\hline 1 & $51(32.7)$ & $39(29.3)$ & $12(52.2)$ & \\
\hline 2 & $58(37.2)$ & $52(39.1)$ & $6(26.1)$ & \\
\hline 3 & $44(28.2)$ & $39(29.3)$ & $5(21.7)$ & \\
\hline Lobular inflammation, n (\%) & & & & 0.372 \\
\hline 0 & $3(1.9)$ & $3(2.2)$ & $0(0.0)$ & \\
\hline 1 & $76(49.0)$ & $68(51.5)$ & $8(34.8)$ & \\
\hline 2 & $70(45.2)$ & $56(42.1)$ & $14(60.9)$ & \\
\hline 3 & $6(3.9)$ & $5(3.8)$ & $1(4.3)$ & \\
\hline Ballooning, n (\%) & & & & $<0.001$ \\
\hline 0 & $38(24.4)$ & $34(25.6)$ & $4(17.4)$ & \\
\hline 1 & $87(55.8)$ & $82(61.6)$ & $5(21.7)$ & \\
\hline 2 & $30(19.2)$ & $16(12.0)$ & $14(60.9)$ & \\
\hline 3 & $1(0.6)$ & $1(0.8)$ & $0(0.0)$ & \\
\hline NASH, n (\%) & & & & 0.012 \\
\hline NAFLD, no NASH & $20(13.0)$ & $20(15.0)$ & $0(0.0)$ & \\
\hline Borderline NASH & $18(11.7)$ & $18(13.5)$ & $0(0.0)$ & \\
\hline
\end{tabular}


Table 1 Continued

\begin{tabular}{|c|c|c|c|c|}
\hline Characteristics & All $(n=156)$ & $\begin{array}{l}\text { No advanced } \\
\text { fibrosis (stage } 0-2) \\
(n=133)\end{array}$ & $\begin{array}{l}\text { Advanced } \\
\text { fibrosis (stage } 3-4) \\
(n=23)\end{array}$ & $P$ value* \\
\hline Definite NASH & $116(75.3)$ & $93(69.9)$ & $23(100.0)$ & \\
\hline NAS, median (IQR) & $4(2)$ & $4(2)$ & $5(2)$ & 0.602 \\
\hline
\end{tabular}

Mean values are provided \pm SD, unless otherwise noted as $n(\%)$ or median and IQR.

* $P$ value determined by comparing characteristics of individuals with advanced fibrosis (fibrosis stage 3-4) and without advanced fibrosis (fibrosis stage $0-2$ ) were evaluated using an independent samples t-test or Wilcoxon-Mann-Whitney test. $\chi^{2}$ test or Fisher's exact test, when appropriate, was used to compare categorical variables. Bold indicates significant $p$ values $<0.05$.

ALT, alanine aminotransferase; Alk P, alkaline phosphatase; AST, aspartate aminotransferase; BMI, body mass index; GGT, gamma-glutamyl transferase; HbA1c, glycated haemoglobin; HDL, high-density lipoprotein; HOMA, Homeostasis Model Assessment; INR, international normalised ratio; LDL, low-density lipoprotein; NAS, NAFLD Activity Score.

\section{Pathway analysis of the top 10 serum metabolites associated with advanced fibrosis}

Among the top 10 metabolites associated with advanced fibrosis, 6 were cholesterol-derived steroid hormones (5alpha-androstan-3beta monosulfate, pregnanediol-3-glucuronide, androsterone sulfate, epiandrosterone sulfate, dehydroisoandrosterone sulfate (DHEA-S), and 5alpha-androstan-3beta disulfate) and were significantly decreased in participants with advanced fibrosis compared with participants without advanced fibrosis. In addition, glycocholate, a primary conjugated bile acid, was significantly increased in participants with advanced fibrosis whereas taurine, an amino acid involved in the bile acid biosynthesis, was decreased. Finally, palmitoleate, a long-chain monounsaturated fatty acid, and the monosaccharide fucose were significantly increased in participants with advanced fibrosis compared with participants without advanced fibrosis (figure 1).

\section{Prediction of advanced fibrosis with a serum metabolites panel compared with FIB-4 index, NFS and clinical variables associated with advanced fibrosis}

Inclusion of the top 10 metabolite panel based on FDR-adjusted $\mathrm{p}$ values yielded an AUROC c-statistic for advanced fibrosis of 0.94 (95\% CI 0.897 to 0.982). The c-statistic for the FIB-4 Index was 0.78 (95\% CI 0.674 to 0.891 ) (figure $2 \mathrm{~A})$. The discriminatory ability for the top 10 serum metabolite panel compared favourably to the FIB-4 index, $\mathrm{p}=0.002$. At a cut-off derived to obtain $90 \%$ sensitivity, specificity remains $79 \%$ in the metabolite model compared with $39 \%$ specificity for FIB-4 index (table 2). Combining the top 10 metabolite panel with the FIB-4 index did not improve the c-statistic, 0.94 (95\% CI 0.896 to 0.983 ), $\mathrm{p}=0.836$. The NFS yielded a diagnostic accuracy of $0.84(95 \%$ CI 0.75 to 0.93 ) for advanced fibrosis but was inferior to the top 10 metabolite panel, $\mathrm{p}=0.017$ and at fixed sensitivity of 90\% only had 59\% specificity. A model derived from factors significantly associated with advanced fibrosis after backward stepwise elimination included alkaline phosphatase, haemoglobin A1c, platelet count and INR and had a c-statistic of 0.84 that was borderline inferior, $p=0.05$, compared with the top 10 metabolite panel. MRI-PDFF was inversely associated with advanced fibrosis with an OR of 0.91 (95\% CI 0.83 to 0.98 ) per $1 \%$ increase in PDFF with a $\mathrm{p}$ value of 0.015 in a subset of patients $(n=117)$. However, it did not significantly improve the discriminatory ability of the top 10 metabolite model ( $c=0.96 \mathrm{vs}$ $\mathrm{c}=0.94, \mathrm{p}=0.54)$.

\section{Validation of the diagnostic performance of the combination of serum metabolites panel}

We further assessed the diagnostic performance of the combination of the 10 serum metabolites identified in the derivation cohort in an independent validation cohort including 142 participants with MRE assessment for advanced fibrosis. They were a mean age of 44.9 years, $73 \%$ non-Hispanic white, predominantly female $(71.9 \%)$ and overweight (mean $\mathrm{BMI}=26.6 \mathrm{~kg} / \mathrm{m}^{2}$ ). Detailed study cohort flow chart is provided in online supplemental figure 1 . The prevalence of advanced fibrosis defined as MRE $\geq 3.63 \mathrm{kPa}$ was $8 \%$ (11 out of 142 ) in the validation cohort. Detailed baseline characteristics of the participants with and without advanced fibrosis compared with participants without advanced fibrosis are presented in table 3.

The application of the 10 serum metabolites identified in the derivation cohort yielded an AUROC c-statistic of $0.94(0.873-$ 1.000) for the detection of advanced fibrosis. The c-statistic for the FIB-4 Index was significantly lower, 0.81 (95\% CI 0.712 to 0.914$)(\mathrm{p}=0.011)$ (figure $2 \mathrm{~B})$ and the $\mathrm{c}$-statistic for NFS was

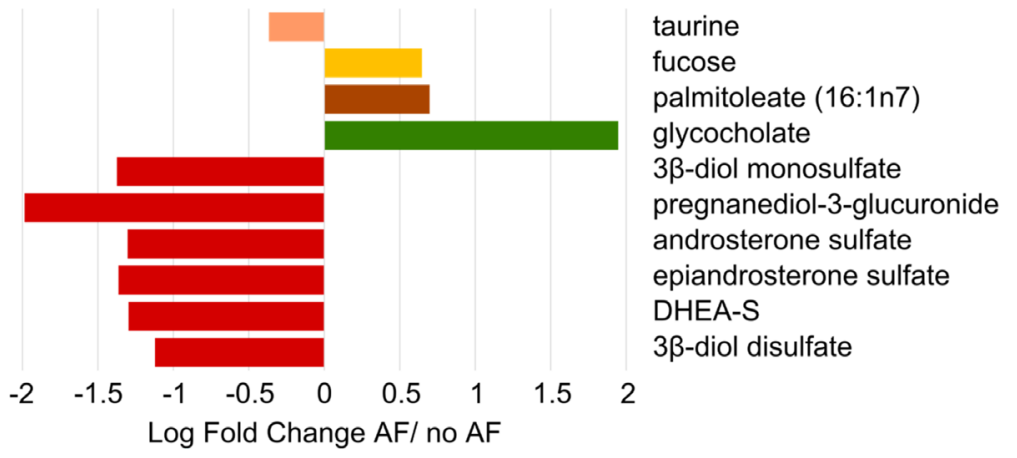

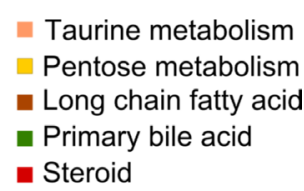

- Steroid

Figure 1 Fold change of the top 10 serum metabolite panel associated with advanced fibrosis The variation of the top 10 serum metabolites including 5alpha-androstan-3beta monosulfate, pregnanediol-3-glucuronide, androsterone sulfate, epiandrosterone sulfate, palmitoleate, dehydroisoandrosterone sulfate, 5alpha-androstan-3beta disulfate, glycocholate, taurine and fucose, coloured based on five subpathways is depicted as log fold change in participants with advanced fibrosis (AF) versus no advanced fibrosis. 
A Derivation biopsy proven cohort

B Validation Twin and Family cohort C Validation biopsy proven NAFLD cohort
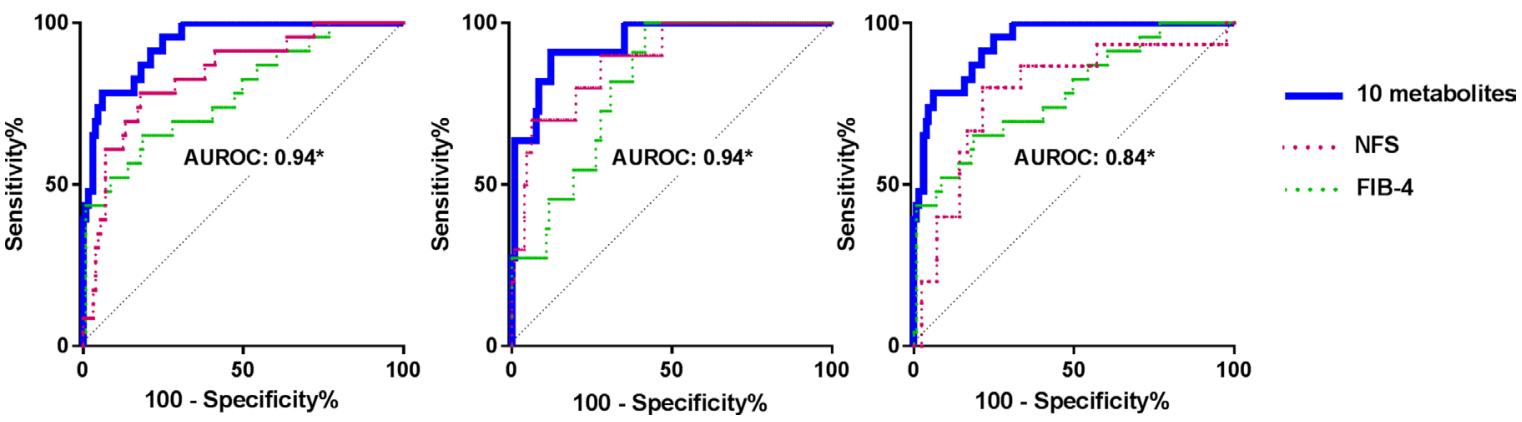

Figure 2 Top 10 metabolite panel has higher area under the receiver operating characteristic curve (AUROC) than NAFLD Fibrosis Score (NFS) and FIB-4 index in derivation and validation cohorts The AUROC of the 10 serum metabolites panel including 5alpha-androstan-3beta monosulfate, pregnanediol-3-glucuronide, androsterone sulfate, epiandrosterone sulfate, palmitoleate, dehydroisoandrosterone sulfate, 5alpha-androstan-3beta disulfate, glycocholate, taurine and fucose, NFS and FIB-4 index for the detection of advanced fibrosis is shown in (A) the derivation biopsy-proven cohort, (B) the validation Twin and Family Cohort and (C) the independent biopsy-proven NAFLD validation cohort. *The AUROC of the 10 serum metabolites was higher than NFS and FIB-4 in the derivation cohort and validation cohorts.

lower but the difference did not reach statistical significance 0.89 (95\% CI 0.786 to 0.987$)(\mathrm{p}=0.32)$. We performed sensitivity analysis in the cohort including only subjects who were not relatives and the diagnostic accuracy of the 10 metabolite panel remained excellent c-statistic $0.96(n=74)$.

We further assessed the diagnostic performance of the combination of the 10 serum metabolites identified in the derivation cohort in an independent validation cohort of 59 patients with biopsy-proven NAFLD (supplemental table 4). The prevalence of advanced fibrosis (stage 3-4) was 31\% (15 out of 59). Two metabolites, 5 alpha-androstan-3beta, 17beta-diol monosulfate and taurine, were either unmeasured or available for less than half of the patients and were excluded. The remaining eight metabolites had good diagnostic accuracy c-statistic 0.84 and compared favourably to FIB-4 index (c-statistic 0.80) and NFS (c-statistic 0.72); however, the differences were not statistically significant (figure 2C).

\section{Serum metabolites associated with NASH histology in the derivation cohort}

Among the 652 serum metabolites analysed, 31 serum metabolites met the FDR adjusted threshold for statistical significant association with presence of NASH. These serum metabolites belonged to four super pathways: amino acid, lipid, nucleotide and peptide (online supplemental table 2). The fold change of the top 10 serum metabolites associated with the presence of NASH is shown in online supplemental figure 2.

\section{Prediction of NASH with serum metabolites panel}

Inclusion of the top 10 metabolite panel based on FDR adjusted $\mathrm{p}$ values yielded an AUROC c-statistic for NASH compared with not NASH histology of 0.79 (95\% CI 0.702 to 0.869 ). Inclusion of all 31 significant FDR adjusted metabolites improved the AUROC c-statistic to 0.89 (95\% CI 0.842 to 0.945 ) (online supplemental figure 3 ). Of the 31 metabolites associated with $\mathrm{NASH}$ in the derivation cohort, only 10 were available and measured in $>50 \%$ of patients in the biopsy-proven NAFLD validation cohort and the c-statistic for the diagnosis of NASH was $\mathrm{c}=0.82$.

\section{Sensitivity analyses}

We have further assessed the association between the level of the serum metabolites included in diagnostic panels and PNPLA3 genotype available in a subgroup of participants from the Twin and Family Cohort $(\mathrm{n}=101)$ and did not observed significant association (online supplemental table 5).

\section{DISCUSSION}

\section{Main findings}

Using a well-characterised derivation cohort of patients with biopsy-proven NAFLD and two validation cohorts of individuals assessed with advanced MRE and liver biopsy, we report that the combination of 10 -serum metabolites can diagnose the presence of advanced fibrosis with greater diagnostic accuracy

Table 2 Diagnostic performance for the detection of advanced fibrosis

\begin{tabular}{|c|c|c|c|c|c|c|}
\hline & AUROC $(95 \% \mathrm{Cl})$ & Sensitivity (\%) & Specificity (\%) & PPV & NPV & $P$ value* \\
\hline 10 metabolites & 0.94 (0.897 to 0.982$)$ & 90 & 79 & 43 & 98 & NA \\
\hline FIB-4 index & 0.78 (0.674 to 0.891$)$ & 90 & 39 & 21 & 96 & 0.002 \\
\hline FIB-4+10 metabolites & 0.94 (0.896 to 0.983 ) & 90 & 79 & 43 & 98 & 0.836 \\
\hline NAFLD Fibrosis Score & 0.84 (0.752 to 0.929$)$ & 90 & 59 & 28 & 97 & 0.017 \\
\hline Clinical predictive score & $0.84(0.724$ to 0.945$)$ & 90 & 37 & 20 & 95 & 0.053 \\
\hline
\end{tabular}

Shown are the specificity, NPV and PPV at the fixed sensitivity of $90 \%$ for the detection of advanced fibrosis.

Bold indicates $p$ values $<0.05$.

${ }^{*} P$ value was determined be comparing the AUROC of the combination of 10 metabolites to the diagnostic test on the same row.

†The score includes platelet count, international normalised ratio, haemoglobin A1c \% and alkaline phosphatase based on backward stepwise elimination of factors associated with advanced fibrosis.

AUROC, area under the receiver operating characteristic curve; NA. not available; NPV, negative predictive value; PPV, positive predictive value, 
Table 3 Baseline characteristics of the validation Twin and Family Cohort

\begin{tabular}{|c|c|c|c|c|}
\hline Characteristics & Overall $(n=142)$ & $\begin{array}{l}\text { No advanced fibrosis } \\
\text { MRE }<3.63 \mathrm{kPa} \\
(\mathrm{n}=131)\end{array}$ & $\begin{array}{l}\text { Advanced fibrosis } \\
\text { MRE } \geq 3.63 \mathrm{kPa} \\
(\mathrm{n}=11)\end{array}$ & $P$ value \\
\hline \multicolumn{5}{|l|}{ Demographics } \\
\hline Age, years & $44.9(19.4)$ & $43.5(19.3)$ & $61.4(11.6)$ & 0.008 \\
\hline Male, $n(\%)$ & $40(28.1)$ & $33(25.2)$ & $7(63.6)$ & 0.012 \\
\hline \multicolumn{5}{|l|}{ Race } \\
\hline White, $n(\%)$ & $113(72.5)$ & $98(74.8)$ & $5(45.5)$ & 0.071 \\
\hline Hispanic, n (\%) & $24(16.9)$ & $20(15.3)$ & $4(36.4)$ & 0.091 \\
\hline BMI $\left(\mathrm{kg} / \mathrm{m}^{2}\right)$ & $26.6(6.2)$ & $26.1(6.0)$ & $33.7(5.3)$ & $<0.001$ \\
\hline \multicolumn{5}{|l|}{ Clinical } \\
\hline Type 2 diabetes, n (\%) & $8(5.6 \%)$ & $6(4.6)$ & $2(18.1)$ & 0.118 \\
\hline \multicolumn{5}{|l|}{ Biological data } \\
\hline Glucose (mg/dL) & $92.2(17.2)$ & $90.7(15.5)$ & $110.6(25.0)$ & 0.026 \\
\hline Insulin (U/L) & $12.1(22.8)$ & $9.2(7.0)$ & $45.8(72.2)$ & $<0.001$ \\
\hline $\mathrm{HbA1C}$ & $5.8(0.5)$ & $5.7(0.5)$ & $6.4(0.7)$ & $<0.001$ \\
\hline HOMA-IR & $2.9(6.1)$ & $2.1(1.9)$ & $12.2(19.1)$ & 0.113 \\
\hline AST (U/L) & $23.9(10.2)$ & $23.0(8.7)$ & $34.6(18.6)$ & 0.009 \\
\hline ALT (U/L & $23.0(15.4)$ & $21.6(13.5)$ & $39.1(25.5)$ & 0.001 \\
\hline Alkaline phosphatase (U/L) & $70.3(23.2)$ & $69.7(22.2)$ & 77.6 (33.5) & 0.280 \\
\hline Total bilirubin (mg/dL) & $0.45(0.22)$ & $0.44(0.21)$ & $0.55(0.25)$ & 0.101 \\
\hline Direct bilirubin (mg/dL) & $0.12(0.04)$ & $0.11(0.03)$ & $0.14(0.05)$ & 0.005 \\
\hline Albumin (g/dL) & $4.55(0.32)$ & $4.6(0.3)$ & $4.5(0.3)$ & 0.433 \\
\hline GGT (U/L) & $25.6(30.2)$ & $20.2(10.6)$ & $88.4(81.2)$ & $<0.001$ \\
\hline Total cholesterol (mg/dL) & $188.5(41.3)$ & $189.0(41.0)$ & $182.1(45.9)$ & 0.594 \\
\hline HDL-cholesterol (mg/dL) & $62.2(17.3)$ & $63.7(17.0)$ & $44.9(8.1)$ & $<0.001$ \\
\hline LDL-cholesterol (mg/dL) & $106.7(35.8)$ & $107.1(35.6)$ & $101.1(39.3)$ & 0.593 \\
\hline Triglycerides (mg/dL) & $97.9(62.0)$ & $90.9(57.1)$ & $179.8(60.6)$ & $<0.001$ \\
\hline White blood cell count $\left(\times 10^{3} / \mu \mathrm{L}\right)$ & $6.0(1.6)$ & $6.0(1.6)$ & $6.3(1.4)$ & 0.587 \\
\hline Haemoglobin (g/dL) & $13.8(2.2)$ & $13.6(1.3)$ & $15.6(6.5)$ & 0.503 \\
\hline Haematocrit (\%) & $40.5(3.4)$ & $40.5(3.3)$ & $39.5(4.0)$ & 0.350 \\
\hline Platelet count $\left(\times 10^{3} / \mu \mathrm{L}\right)$ & $255.6(52.6)$ & $258.8(51.3)$ & $217.2(55.1)$ & 0.011 \\
\hline INR & $1.04(0.18)$ & $1.0(0.2)$ & $1.0(0.8)$ & 0.783 \\
\hline Ferritin (ng/mL) & $88.4(88.6)$ & $84.6(70.5)$ & $137.2(216.4)$ & 0.463 \\
\hline \multicolumn{5}{|l|}{ Imaging } \\
\hline MRI-PDFF (\%) & $4.8(5.5)$ & $3.9(4.1)$ & $15.1(8.9)$ & $<0.001$ \\
\hline MRE (kPa) & $2.4(0.8)$ & $2.2(0.4)$ & $4.7(1.0)$ & $<0.001$ \\
\hline \multicolumn{5}{|l|}{ Clinical prediction rule } \\
\hline FIB-4 & $0.97(0.58)$ & $0.91(0.51)$ & $1.7(0.83)$ & 0.001 \\
\hline NAFLD Fibrosis Score & $-2.523(1.401)$ & $-2.682(1.274)$ & $-0.455(1.392)$ & $<0.001$ \\
\hline
\end{tabular}

Mean values are provided with the SD in parenthesis, unless otherwise noted as $n(\%)$. Differences between individuals with and without advanced fibrosis were evaluated with $t$ tests or the Wilcoxon-Mann-Whitney test for continuous variables and the $\chi^{2}$ or Fisher's exact test for categorical variables. Bold indicates significant $p$ values $\leq 0.05$.

ALT, alanine aminotransferase; AST, aspartate aminotransferase; BMI, body mass index; GGT, gamma-glutamyl transpeptidase; HOMA-IR, Homeostatic Model Assessment-Insulin Resistance; HbA1c, glycated haemoglobin; HDL, high-density lipoprotein; INR, international normalised ratio; LDL, low-density lipoprotein; MRI-PDFF, MRI protein density fat fraction; MRE, MRI elastography.

than the FIB-4 index. This proof-of-concept study demonstrates that a non-invasive blood-based diagnostic test can provide excellent performance characteristics for the detection of advanced fibrosis, which is the strongest predictor of liver-related mortality. ${ }^{3-5}$ In addition, we also report an excellent diagnostic accuracy of a combination of 31-serum metabolites panel for the detection of NASH in the patients with biopsy-proven NAFLD. However, further studies are needed to confirm the diagnostic performance of this novel combination of metabolites for the detection of NASH in independent cohorts. These novel data have potential important implication for the non-invasive screening of advanced fibrosis and NASH in patients with NAFLD which remains a major unmet need in the field.

\section{In context of published literature}

Several studies have evaluated combinations of clinical and laboratory variables for the detection of advanced fibrosis in NAFLD and were recently reviewed by Younossi et al and Vilar-Gomez et $\mathrm{al}^{79}$ Currently, the most commonly used non-invasive tests to diagnose advanced fibrosis in NAFLD classify approximately $30 \%$ of patients as indeterminate, leaving the need for a diagnostic test to better characterise disease severity unmet. ${ }^{8}{ }^{9}$ While a recent meta-analysis demonstrated that the FIB-4 index has superior performance compared with the NAFLD Fibrosis Score and BARD score, ${ }^{38}$ these clinical prediction rules lack the specificity to accurately diagnose patients with advanced fibrosis. By increasing the specificity, as seen with the proposed metabolomics-based approach, the number of 
false-positive patients that would likely proceed to liver biopsy can be dramatically reduced. Application of the top 10 metabolite panel yielded only 28 false positives compared with 54 with NFS and 81 with FIB-4 in the derivation cohort of 156 patients. Combinations of serum markers and clinical variables are prone to overestimate disease severity in the elderly. ${ }^{39} \mathrm{~A}$ metabolomics-based approach does not rely on clinical factors but rather on biological mechanisms, which prevents it from having poor performance in subpopulations including the elderly and patients with type 2 diabetes. Multiple groups have recently reported that a combination of serum metabolites could be a useful biomarker to differentiate steatosis from $\mathrm{NASH}^{1140}$; however, the diagnostic performance of a combination of serum metabolites for the detection of advanced fibrosis has not been reported yet.

The top 10 serum metabolites significantly associated with the presence of advanced fibrosis in the derivation cohort belong to pathways and subpathways with known associations with the severity of NAFLD. Interestingly, 6 of these 10 metabolites are cholesterol-derived precursors of steroid hormones and were significantly lower in participants with advanced fibrosis compared with participants without advanced fibrosis. The reduction in these cholesterol-derived steroid hormones may result from decreased synthesis and suggests a strong association between regulation of this pathway and the presence of advanced fibrosis in NAFLD. Indeed, reductions in DHEA-S have been previously associated with histologically advanced NAFLD and alterations in these pathways may mediate NAFLD pathogenesis through changes in insulin sensitivity, susceptibility to oxidative stress and/or stimulation of fibrosis. ${ }^{16}$

Furthermore, impaired bile acid metabolism has been reported to potentially contribute to the pathophysiology of NAFLD. ${ }^{41}$ In line with these findings, the primary conjugated bile acid, glycocholate, was one of the top 10 metabolites identified and was significantly increased in presence of advanced fibrosis. Previous studies have reported a higher level of glycocholate in patients with NAFLD including patients with significant fibrosis (stage $\geq 2$ ). ${ }^{18} 42$ In addition, a significant decrease in the level of taurine was associated with advanced fibrosis. Taurine is an amino acid associated with bile acid conjugation in the hepatocyte. In mice, taurine deficiency induces the development of liver fibrosis, ${ }^{43}$ and studies suggest that its antioxidant properties could have a protective effect on experimental models of fibrosis in rodents. ${ }^{446}$

Higher serum palmitoleate was associated with advanced fibrosis and has previously been reported to be increased in patients with NAFLD. ${ }^{10}$ This long-chain monounsaturated fatty acid is produced during de novo lipogenesis. Finally, increase in the serum level of the monosaccharide fucose has not been previously reported in patients with advanced fibrosis. However, emerging data suggest that fucosylated glycan could be potential biomarker of advanced NAFLD. ${ }^{47}$

Even though the causal role of the metabolites identified could not be determined in this cross-sectional study design, the levels of these metabolites were associated with the presence of advanced fibrosis and likely reflect alterations in key pathways associated with the progression of the disease. Hence, a combination of these metabolites is expected to be a more direct measure of disease activity than previous classic indirect markers such as ALT, AST and platelet count. In addition, the combination of several metabolites related to different pathways decreases the likelihood of potential cofounding related to gender, age or metabolic conditions including obesity and type 2 diabetes.

\section{Strengths and limitations}

There are several notable strengths in this study including the use of a derivation and two independent validation cohorts that were prospectively recruited and well-characterised using liver biopsy or advanced MRI of the liver. In addition, conditions such as excessive alcohol use, steatogenic medications, viral hepatitis and secondary causes of steatosis were systematically excluded. We validated our findings in two cohorts, one of which used the most accurate non-invasive modality for the assessment of hepatic fibrosis ${ }^{48}$ and the other had biopsy-proven NAFLD. However, we acknowledge the following limitations of the study. Potential confounding factor such as diet were not captured in the study and therefore could not be excluded. We were limited in our validation of the diagnostic performance of the combination of 31 metabolites for the diagnosis of NASH. In addition, the cross-sectional study design only allowed for metabolomic profiling associated with the presence of advanced fibrosis or NASH at one time point. Further longitudinal studies are needed to determine if this new biomarker can longitudinally detect changes in liver fibrosis and NASH. Furthermore, PNPLA3 genotype, which has been shown to be an independent predictor of $\mathrm{NASH}^{49}$ and strongly influences the liver lipidome, ${ }^{50}$ was only available in a subset of the study population limiting our ability to explore its effect on the predictive value of the metabolite panel. Finally, the baseline characteristics of the validation cohorts were different from the derivation cohort. In addition, the MRE validation cohort included participants with and without NAFLD and therefore the pre-test probability of advanced fibrosis was lower compared with the derivation cohort, which included only NAFLD participants. Although these differences typically decrease the diagnostic performance of the test on the validation cohort, the findings remained robust and the diagnostic accuracy was confirmed.

\section{Implications for future study}

In this proof-of-concept study using three independent and well-characterised cohorts, we demonstrate that a serum metabolite panel can accurately predict the presence of advanced fibrosis and outperforms the most frequently used clinical prediction rules. Further validation of these findings in separate cohorts and evaluation of longitudinal changes in serum metabolites to assess disease progression or treatment response are warranted.

Acknowledgements This work was presented in abstract form at The Liver Meeting 2018 (San Francisco, California, USA).

Contributors CC: study concept and design, analysis and interpretation of data, drafting of the manuscript, critical revision of the manuscript, approved final submission. VA: study concept and design, analysis and interpretation of data, drafting of the manuscript, critical revision of the manuscript, approved final submission. PP: collection, analysis and interpretation of data, critical revision of the manuscript, approved final submission C-H: statistical analysis, critical revision of the manuscript, approved final submission SB: data collection, critical revision of the manuscript, approved final submission. MM: data collection, critical revision of the manuscript, approved final submission SS: data collection, critical revision of the manuscript, approved final submission Claire Faulkner: data collection, critical revision of the manuscript, approved final submission MAV: data collection, critical revision of the manuscript, approved final submission ER: patient visits, critical revision of the manuscript, approved final submission LR: patient visits, critical revision of the manuscript, approved final submission DAB: study concept and design, critical revision of the manuscript, study supervision, approved final submission CBS: analysis and interpretation of data, drafting of the manuscript, critical revision of the manuscript, obtained funding, study supervision, approved final submission AJS: critical revision of the manuscript, approved final submission RL: study concept and design, analysis and interpretation of data, drafting of the manuscript, critical revision of the manuscript, obtained funding, study supervision, approved final submission

Funding RL is supported in part by the American Gastroenterological Association (AGA) Foundation - Sucampo - ASP Designated Research Award in Geriatric Gastroenterology and by a T. Franklin Williams Scholarship Award; Funding provided by: Atlantic Philanthropies, Inc, the John A. Hartford Foundation, OM, the Association of Specialty Professors, and the American Gastroenterological Association and grant K23-DK090303. CS and RL serve as co-PIs on the grant R01-DK106419. The content is solely the responsibility of the authors and does not necessarily represent the official views of the NIH. VA is supported by the AASLD Foundation Clinical 
and Translational Research Award. CC is supported by grants from the Société Francophone du Diabète (SFD), the Philippe Foundation and Monahan Foundation under the Fulbright program.

Competing interests CBS consults, advises and is on the speakers' bureau for Bayer. He received grants from GE Healthcare

Patient consent Not required.

Ethics approval This study was Health Insurance Portability and Accountability Act (HIPAA) compliant and was approved by the UCSD Institutional Review Board 111282.

Provenance and peer review Not commissioned; externally peer reviewed.

\section{REFERENCES}

1 Loomba R, Sanyal AJ. The global NAFLD epidemic. Nat Rev Gastroenterol Hepatol 2013;10:686-90.

2 Younossi ZM, Koenig AB, Abdelatif D, et al. Global epidemiology of nonalcoholic fatty liver disease-Meta-analytic assessment of prevalence, incidence, and outcomes. Hepatology 2016:64:73-84.

3 Dulai PS, Singh S, Patel J, et al. Increased risk of mortality by fibrosis stage in nonalcoholic fatty liver disease: Systematic review and meta-analysis. Hepatology 2017:65:1557-65.

4 Angulo P, Kleiner DE, Dam-Larsen S, et al. Liver fibrosis, but no other histologic features, is associated with long-term outcomes of patients with nonalcoholic fatty liver disease. Gastroenterology 2015;149:389-97.

5 Ekstedt $\mathrm{M}$, Hagström H, Nasr P, et al. Fibrosis stage is the strongest predictor for disease-specific mortality in NAFLD after up to 33 years of follow-up. Hepatology 2015:61:1547-54

6 Loomba R. Role of imaging-based biomarkers in NAFLD: Recent advances in clinical application and future research directions. J Hepatol 2018;68:296-304.

7 Younossi ZM, Loomba R, Anstee QM, et al. Diagnostic Modalities for Non-alcoholic Fatty Liver Disease (NAFLD), Non-alcoholic Steatohepatitis (NASH) and Associated Fibrosis. Hepatology 2017

8 McPherson S, Stewart SF, Henderson E, et al. Simple non-invasive fibrosis scoring systems can reliably exclude advanced fibrosis in patients with non-alcoholic fatty liver disease. Gut 2010;59:1265-9.

9 Vilar-Gomez E, Chalasani N. Non-invasive assessment of non-alcoholic fatty liver disease: Clinical prediction rules and blood-based biomarkers. J Hepatol 2018:68:305-15.

10 Puri P, Wiest MM, Cheung 0 , et al. The plasma lipidomic signature of nonalcoholic steatohepatitis. Hepatology 2009;50:1827-38.

11 Alonso C, Fernández-Ramos D, Varela-Rey M, et al. Metabolomic Identification of Subtypes of Nonalcoholic Steatohepatitis. Gastroenterology 2017;152:1449-61.

12 Barr J, Caballería J, Martínez-Arranz I, et al. Obesity-dependent metabolic signatures associated with nonalcoholic fatty liver disease progression. J Proteome Res 2012;11:2521-32

13 Mardinoglu A, Agren R, Kampf C, et al. Genome-scale metabolic modelling of hepatocytes reveals serine deficiency in patients with non-alcoholic fatty liver disease. Nat Commun 2014:5:3083.

14 Kaikkonen JE, Würtz P, Suomela E, et al. Metabolic profiling of fatty liver in young and middle-aged adults: Cross-sectional and prospective analyses of the Young Finns Study. Hepatology 2017;65:491-500.

15 Sookoian S, Puri P, Castaño GO, et al. Nonalcoholic steatohepatitis is associated with a state of betaine-insufficiency. Liver Int 2017;37:611-9.

16 Charlton M, Angulo P, Chalasani N, et al. Low circulating levels of dehydroepiandrosterone in histologically advanced nonalcoholic fatty liver disease. Hepatology 2008;47:484-92.

17 Jin R, Banton S, Tran VT, et al. Amino acid metabolism is altered in adolescents with nonalcoholic fatty liver disease-an untargeted, high resolution metabolomics study. $J$ Pediatr 2016:172:14-19.

18 Puri P, Daita K, Joyce A, et al. The presence and severity of nonalcoholic steatohepatitis is associated with specific changes in circulating bile acids. Hepatology 2017

19 Polyzos SA, Kountouras J, Tsatsoulis A, et al. Sex steroids and sex hormone-binding globulin in postmenopausal women with nonalcoholic fatty liver disease. Hormones 2013;12:405-16.

20 Koehler E, Swain J, Sanderson S, et al. Growth hormone, dehydroepiandrosterone and adiponectin levels in non-alcoholic steatohepatitis: an endocrine signature for advanced fibrosis in obese patients. Liver Int 2012:32:279-86.

21 Loomba R, Quehenberger O, Armando A, et al. Polyunsaturated fatty acid metabolites as novel lipidomic biomarkers for noninvasive diagnosis of nonalcoholic steatohepatitis. J Lipid Res 2015;56:185-92.

22 Park CC, Nguyen P, Hernandez C, et al. Magnetic resonance elastography vs transient elastography in detection of fibrosis and noninvasive measurement of steatosis in patients with biopsy-proven nonalcoholic fatty liver disease. Gastroenterology 2017; 152:598-607.
23 Caussy C, Hsu C, Lo MT, et al. Link between gut-microbiome derived metabolite and shared gene-effects with hepatic steatosis and fibrosis in NAFLD. Hepatology 2018:918-32.

24 Caussy C, Chen J, Alquiraish MH, et al. Association between obesity and discordance in fibrosis stage determination by magnetic resonance vs transient elastography in patients with nonalcoholic liver disease. Clin Gastroenterol Hepatol 2018;16:1974-82.

25 Ajmera V, Park CC, Caussy C, et al. Magnetic resonance imaging proton density fat fraction associates with progression of fibrosis in patients with nonalcoholic fatty liver disease. Gastroenterology 2018;155:307-10.

26 Zarrinpar A, Gupta S, Maurya MR, et al. Serum microRNAs explain discordance of non-alcoholic fatty liver disease in monozygotic and dizygotic twins: a prospective study. Gut 2016;65:1546-54.

27 Loomba R, Schork N, Chen CH, et al. Heritability of hepatic fibrosis and steatosis based on a prospective twin study. Gastroenterology 2015;149:1784-93.

28 Cui J, Chen CH, Lo MT, et al. Shared genetic effects between hepatic steatosis and fibrosis: a prospective twin study. Hepatology 2016;64:1547-58.

29 Caussy C, Soni M, Cui J, et al. Nonalcoholic fatty liver disease with cirrhosis increases familial risk for advanced fibrosis. J Clin Invest 2017:127:2697-704.

30 Evans AM, DeHaven CD, Barrett T, et al. Integrated, nontargeted ultrahigh performance liquid chromatography/electrospray ionization tandem mass spectrometry platform for the identification and relative quantification of the smallmolecule complement of biological systems. Anal Chem 2009;81:6656-67.

31 Dehaven CD, Evans AM, Dai $\mathrm{H}$, et al. Organization of GC/MS and LC/MS metabolomics data into chemical libraries. J Cheminform 2010;2:9.

32 Permutt Z, Le TA, Peterson MR, et al. Correlation between liver histology and novel magnetic resonance imaging in adult patients with non-alcoholic fatty liver disease - MRI accurately quantifies hepatic steatosis in NAFLD. Aliment Pharmacol Ther 2012;36:22-9

33 Patel NS, Peterson MR, Brenner DA, et al. Association between novel MRI-estimated pancreatic fat and liver histology-determined steatosis and fibrosis in non-alcoholic fatty liver disease. Aliment Pharmacol Ther 2013;37:630-9.

34 Kleiner DE, Brunt EM, Van Natta M, et al. Design and validation of a histological scoring system for nonalcoholic fatty liver disease. Hepatology 2005;41:1313-21.

35 Sterling RK, Lissen E, Clumeck N, et al. Development of a simple noninvasive index to predict significant fibrosis in patients with HIV/HCV coinfection. Hepatology 2006:43:1317-25.

36 Angulo P, Hui JM, Marchesini G, et al. The NAFLD fibrosis score: a noninvasive system that identifies liver fibrosis in patients with NAFLD. Hepatology 2007:45:846-54

37 DeLong ER, DeLong DM, Clarke-Pearson DL. Comparing the areas under two or more correlated receiver operating characteristic curves: a nonparametric approach. Biometrics 1988:44:837-45.

38 Sun W, Cui H, Li N, et al. Comparison of FIB-4 index, NAFLD fibrosis score and BARD score for prediction of advanced fibrosis in adult patients with non-alcoholic fatty liver disease: A meta-analysis study. Hepatol Res 2016;46:862-70.

39 McPherson S, Hardy T, Dufour JF, et al. Age as a confounding factor for the accurate non-invasive diagnosis of advanced NAFLD fibrosis. Am I Gastroenterol 2017:112:740-51.

40 Zhou Y, Orešič M, Leivonen M, et al. Noninvasive detection of nonalcoholic steatohepatitis using clinical markers and circulating levels of lipids and metabolites. Clin Gastroenterol Hepatol 2016;14:1463-72.

41 Chávez-Talavera 0, Tailleux A, Lefebvre P, et al. Bile acid control of metabolism and inflammation in obesity, type 2 diabetes, dyslipidemia, and nonalcoholic fatty liver disease. Gastroenterology 2017;152:1679-94.

42 Kalhan SC, Guo L, Edmison J, et al. Plasma metabolomic profile in nonalcoholic fatty liver disease. Metabolism 2011;60:404-13.

43 Warskulat $U$, Borsch $E$, Reinehr $\mathrm{R}$, et al. Taurine deficiency and apoptosis: findings from the taurine transporter knockout mouse. Arch Biochem Biophys 2007:462:202-9.

44 Miyazaki T, Bouscarel B, Ikegami T, et al. The protective effect of taurine against hepatic damage in a model of liver disease and hepatic stellate cells. Adv Exp Med Biol 2009;643:293-303.

45 Yalçinkaya S, Unlüçerçi Y, Giriș M, et al. Oxidative and nitrosative stress and apoptosis in the liver of rats fed on high methionine diet: protective effect of taurine. Nutrition 2009;25:436-44

46 Refik Mas M, Comert B, Oncu K, et al. The effect of taurine treatment on oxidative stress in experimental liver fibrosis. Hepatol Res 2004;28:207-15.

47 Kamada Y, Ono M, Hyogo H, et al. A novel noninvasive diagnostic method for nonalcoholic steatohepatitis using two glycobiomarkers. Hepatology 2015:62:1433-43

48 Loomba R, Wolfson T, Ang B, et al. Magnetic resonance elastography predicts advanced fibrosis in patients with nonalcoholic fatty liver disease: a prospective study. Hepatology 2014;60:1920-8.

49 Hyysalo J, Männistö VT, Zhou Y, et al. A population-based study on the prevalence of NASH using scores validated against liver histology. J Hepatol 2014;60:839-46.

50 Luukkonen PK, Zhou Y, Sädevirta S, et al. Hepatic ceramides dissociate steatosis and insulin resistance in patients with non-alcoholic fatty liver disease. J Hepatol 2016;64:1167-75 